\title{
Optical Inter-Satellite Communication: the Alphasat and Sentinel-1A in-orbit experience
}

\author{
Edoardo Benzi \\ European Space Agency, ESTEC, Noordwijk, The Netherlands \\ Ian Shurmer, Nicola Policella \\ European Space Agency, ESOC, Darmstadt, Germany \\ Daniel Troendle \\ TESAT Spacecom GmbH \& Co.KG, Backnang, Germany \\ Michael Lutzer \\ DLR, Bonn, Germany \\ Sven Kuhlmann \\ DLR-GSOC, Oberpfaffenhofen, Germany \\ Mark James \\ Inmarsat plc, London, United Kingdom
}

Since late 2013, the Inmarsat operated Alphasat GEO communication satellite has been providing a reliable platform for the experimental activities of its 4 hosted Technical Demonstration Payloads (TDPs), procured and operated by ESA. TDP1 was developed by TESAT Spacecom for DLR, with the objective to demonstrate and characterise a LEO to GEO optical communications link. TDP1 is able to receive observation data from a lower orbit (LEO) spacecraft thanks to its Laser Communication Terminal, and route the data to ground via a Ka-band RF link performed by its own Ka-band System.

In the absence of a counter-terminal aloft, the activities concentrated initially on characterising and optimising the terminal performances using the ESA Optical Ground Station in Tenerife. With the launch in April 2014 of Sentinel-1A in its sun-synchronous orbit, an in-flight companion for the ASA terminal was made available. Operated by ESA on behalf of the European Commission as part of the Copernicus program, S1A performs its earth observation mission thanks to a Synthetic Aperture Radar, and carries on board an Optical Communication Payload, based on another TESAT Laser Communication Terminal.

As a proof of concept for the upcoming European Data Relay Satellites system (EDRS), an Optical Inter-Satellite Link demonstration campaign started in September 2014. Using an incremental approach that led to the first data transmission from Sentinel $1 \mathrm{~A}$ in November 2014, the campaign was completed in December 2014, with results well beyond expectation in terms of link performances.

A subsequent "Experimentation Phase" aiming at the characterisation of the paired terminal performances over its operational envelope has been going on since the beginning of 2015. In the meanwhile, the launch of Sentinel $2 A$ provided the second LEO counter-terminal for TDP1.

Beyond the technological complexity of the laser communication terminal and the outstanding results obtained, the exercise presented some remarkable operational challenges. Planning, scheduling and executing a link implies coordination of the activities of the two missions engineering and operations systems and teams, with the necessary involvement of the laser terminal provider team expertise.

In addition to providing the ultimate demonstration of the technology underlying the EDRS, the Alphasat and Sentinel 1A experiences have thus provided an important opportunity to test the system operations in a realistic scenario, and gain valuable experience to be put to fruition for the EDRS GS and operations segment. 


\section{Introduction}

High-resolution images taken by Earth Observation satellites in Low Earth Orbits (LEO) are more-and-more becoming essential in support of civil and disaster management tasks and applications. Having the right data at the right time can be the difference between success and failure and may even be vital, for example, for coordination of rescue and aid teams following natural disasters such as earthquakes. Earth Observation satellites mostly use direct RF links to deliver their images and recorded data to ground (Figure 1, top).

For images requiring high downlink data-rates or rapid delivery, the LEO satellite capability is improved by increasing the number of global ground receive stations. This increases the number of visibility arcs, but increases ground-segment complexity \& cost. It is also limited geographically to land-based
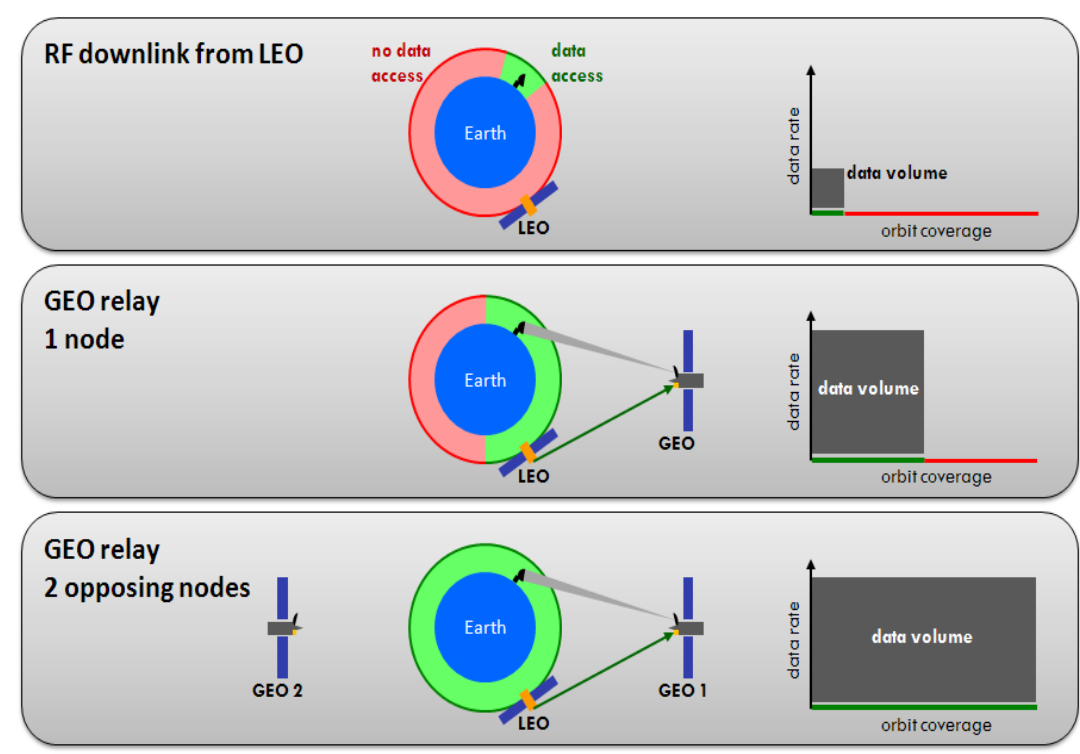

Figure 1: RF downlink from LEO compared with GEO relay: orbit coverage and data volume (schematic) locations and further restrictions such as stringent ITU interference regulations and geo-political considerations.

Optical GEO-relay networks offer the possibility to greatly enhance data return by complementing the typical direct to earth scenario. They permit both high data rates, increased orbit coverage and enable near-real-time data access. Using a GEO-relay, the LEO satellite transmits data to a GEO satellite which receives the data and immediately forwards them to ground. For the LEO-GEO inter-satellite segment, a laser link is the ideal solution offering secure point-to-point communication and superior data rates not subjected to ITU frequency or bandwidth regulations.

Although optical communication has been proven possible via a series of space to ground links, the downlink leg of the "Intersatellite Communication" concept relies on a traditional high-performance RF spot beam link architecture. This avoids the main constraint of optical communication (need of a clear line of sight), with the possible use of multiple RF channels to ensure the end to end system high data throughput. The downlink from GEO to ground ideally uses a high-performance RF spot beam.

A GEO relay with one single GEO node already provides hemispherical coverage. A second GEO node located at the opposite GEO position will enable nearly global coverage (Figure 1, center and bottom). Waiting for the next satellite ground pass is no longer required, and data can be made available in quasi-real-time with only latency times of GEO transmission applying. The ground station can be at any convenient location.

The Key technology for the implementation of the Inter-Satellite Link data transfer system presented here is the Laser Communication Terminal used for the Optical Inter-Satellite Link (OISL), briefly presented in the next section.

\section{The Laser Communication Terminal (LCT)}

TESAT Laser Communication Terminals provide full-duplex inter-satellite communication at $1.8 \mathrm{Gbps}$. For comparison, this data rate equals to USB-3.0 consumer electronics standard. The existing system design covers both LEO-GEO as well as GEO-GEO links with up to $72000 \mathrm{~km}$ link distance at full data rate. The optical links are using infra-red laser light at $1064 \mathrm{~nm}$ wavelength. Coherent BPSK homodyne reception ensures robustness 
against sun blinding and jamming. A $7.2 \mu$ rad beam divergence angle, comparing to a beam diameter of $320 \mathrm{~m}$ at LEO-GEO distance, enables "stealth" communication and prevents interception of the link by other parties.

The LCT contains all functions for data handling (lasers, modulator, de-modulator, fiber amplifier and receiver), all optical elements for beam shaping and steering, and all electronics necessary for mechanism command and control, trajectory calculation and link quality measurements. After loading a link scenario into the LCT internal controller ( $\sim 4$ Kbytes / link), the LCT is able to autonomously manage the pointing, acquisition and data transfer.

The LCT uses a two-axis gimbal for coarse beam pointing and two small two axis mirrors for fine pointing and pointahead angle control. A clever algorithm allows to use the beam steering mechanisms for spatial acquisition, saving size, weight, and power that would otherwise be needed for an additional beacon laser.

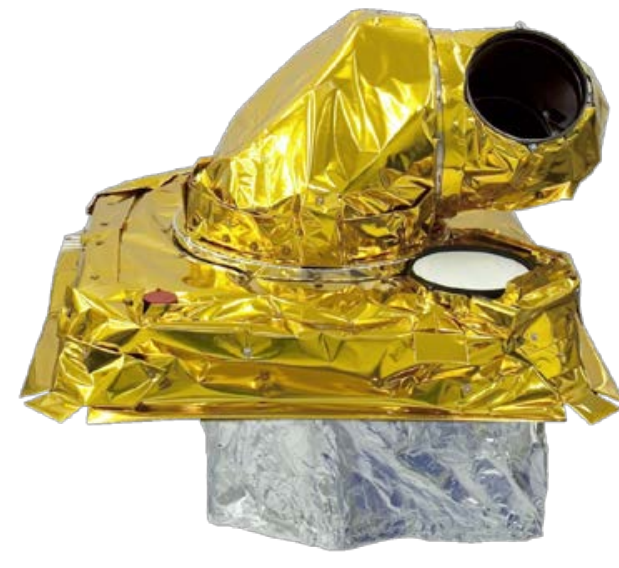

Figure 2: Laser Communication Terminal

The LCT is a single unit with a four-footed quasi-isostatic mechanical mounting interface allowing for fast and easy integration onboard spacecraft. Thermal isolation from spacecraft by a dedicated radiator facilitates thermal

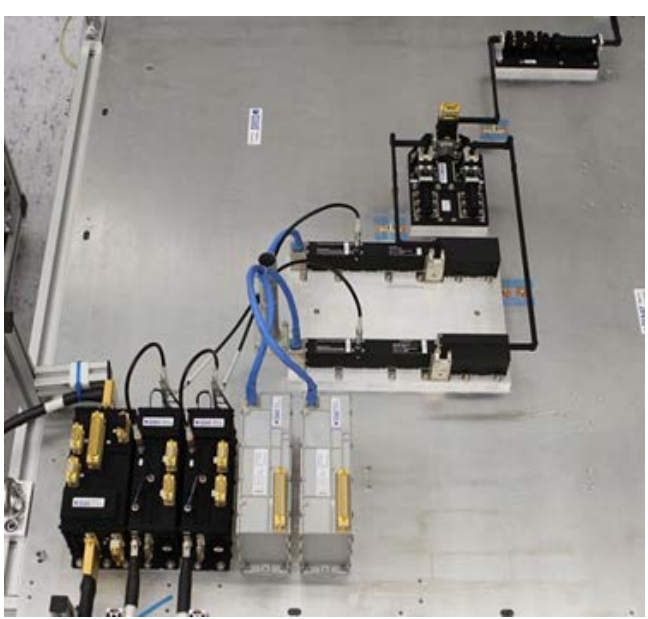

Figure 3: TDP1 Ka-band transmitter mounted on test panel control and stabilization of the laser-optical components. The standardized modular design and interfaces make the LCT suitable for embarkation on basically all types of LEO and GEO spacecraft and allows for adaptation to various scenarios and customer applications. Design lifetime is more than 15 years in GEO orbit. The system budgets and margins validated in orbit and the flexible design allow for both simplified LEO-terminals ('LEO-light') and for increased data rates up to 7.2 Gbps in future designs.

\section{The Ka-Band Transmitter}

In the case of Alphasat, the data received by optical intersatellite links is relayed to ground by means of a Ka-band transmitter operating at $26.5 \mathrm{GHz}$ carrier frequency. The modular system consists of the following: a Digital Switch Matrix (DSM) unitthat serves as the data interface to the LCT and allows data channel switching/routing; two RF chains in cold redundancy with each other; a modulator; a TWTA (EPC+TWT); and an RF isolator. A waveguide switch routes the RF signal through a bandpass and highpass filter towards a

Ka-band antenna.

As a special feature unique to TDP1, the LCT can use the Ka-band downlink to continuously transmit its complete set of internal telemetries with sample rates up to $25 \mathrm{kHz}$, allowing a large variety of experiments and detailed analysis of internal processes.

\section{Optical Inter-Satellite Link Execution Concept}

In order to perform an Optical Inter-Satellite Link, the two laser terminals involved need to share exact relative position information, as well as being precisely synchronised. The very high directionality of the laser beam also implies that the two terminals' optical axes need to be accurately aligned, translating in a RT accurate knowledge of the hosting spacecraft's attitude.

The relative position is provided to each of the two terminals via ground-calculated Chebychev polynomials providing the counter-terminal relative trajectory valid for the link time-window. This is transformed on-board 
by the LCT into the necessary pointing information used during the acquisition phase as well as for the rest of the link. Time synchronisation relies on off the shelf, commonly used means of spacecraft time correlation (ie, notably, use of GPS time), and systematic OBT correction. As a matter of fact, the required time accuracy is generally well within the standard OBT accuracy used by both LEO and GEO missions. The attitude knowledge is granted directly on-board, by establishing a direct data interface for the LCT electronics with the hosting satellite Attitude Control System, whereas the time-resolution and precision of commonly used star-tracker based ACS's satisfy the LCT operations' requirements.

The link itself is carried out in five main phases: (1) Preparation, (2) Spatial Acquisition, (3) Tracking and Frequency acquisition, (4) Communication, and (5) Link termination.

\section{Link Preparation}

At a suitable time before link start time, the two terminals independently prepare for the operations by unparking (in case they have been previously parked, see (5) below) and reaching a predetermined "link start"
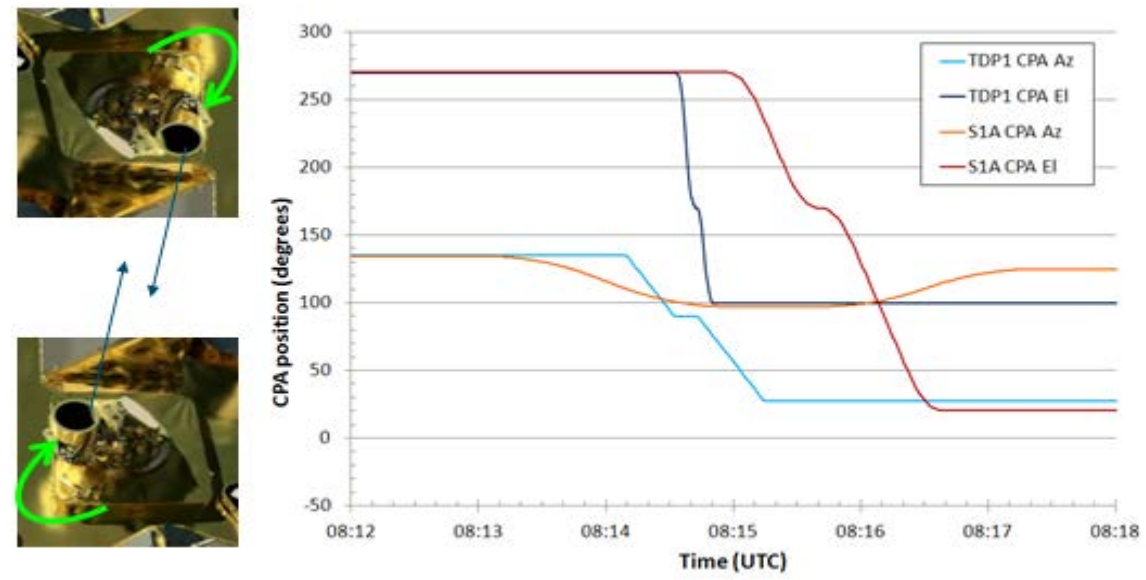

Figure 4: LCT preparation for a link - CPA movement to link start position position, close to the calculated initial point of mutual acquisition (fig. 4), and of the "link trajectory”.

During this phase, the two terminals' Coarse Pointing Assembly (CPA) move to prealign the telescopes' optical axes. Figure 6 shows the actual telemetry of the Azimuth and Elevation angles change for the Alphasat TDP1 and Sentinel-1A OCP CPA's in this phase.

\section{Spatial Acquisition}

Shortly prior to the start of the link, CPAs begin to follow the pre-calculated trajectory, and at the start time of the link (T0) the beaconless spatial “Coarse Acquisition” phase starts (see fig. 5). In the first phase of the coarse acquisition (phase 1), the "Master" terminal initiates spiraling its TX laser beam around the target trajectory line of sight with a total uncertainty cone large enough to score hits at the counter terminal ("Slave”). The "Slave” LCT detects the laser signal with a four-quadrant sensor system and coarsely aligns its optical path in accordance to the detected hits density distribution. In the subsequent "phase 2", the roles are inverted, and the "Slave" terminal
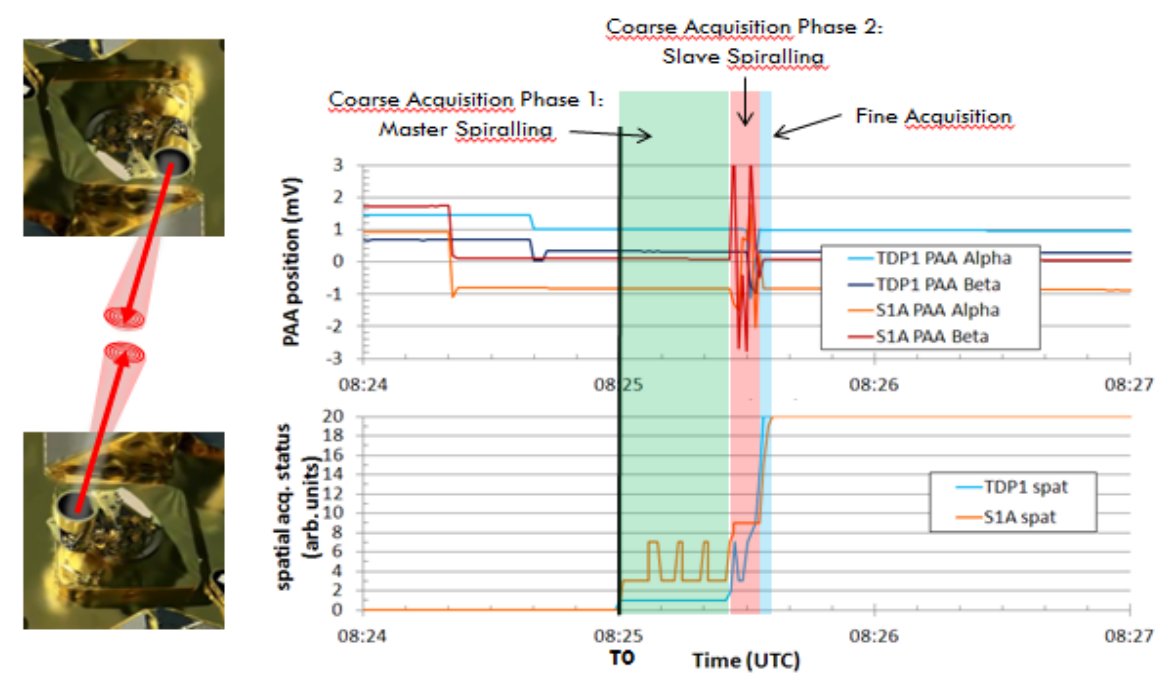

Figure 5: Spatial Acquisition: coarse and fine acquisition sub-phases 
performs the same spiraling in the direction of highest hits density. The process is iterated in the "Fine Acquisition" phase, where the alignment is refined until both LCTs are aligned better than $10 \mu \mathrm{rad}$ about the mutual line of sight, to bring the signal onto the "tracking sensor", and achieve and maintain active tracking.

\section{Tracking and Frequency Acquisition}

Once the alignment of the optical axes is completed, the two terminals start actively tracking each other and perform frequency acquisition, locking their local oscillator laser coherently onto the received signal by an optical phase locked loop.

Fig. 6 shows the Tracking Sensor signal for the two terminals, as well as the "frequency status" (from unlocked to optical phase
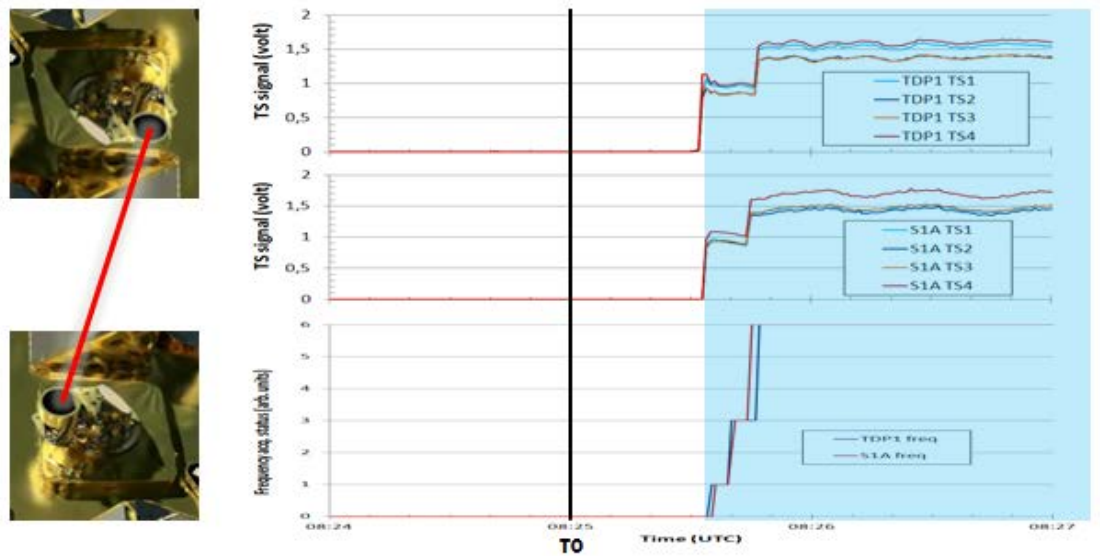
lock).

Figure 6: Tracking and Frequency acquisition phase

\section{Communication}

Once the frequency acquisition is complete, the two terminals start exchanging data modulated (BPSK) on the optical signal. During this phase, active tracking of the counter-terminal signal is performed, with the "Point

Ahead Angle” assembly providing the necessary compensation for the

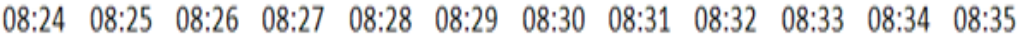

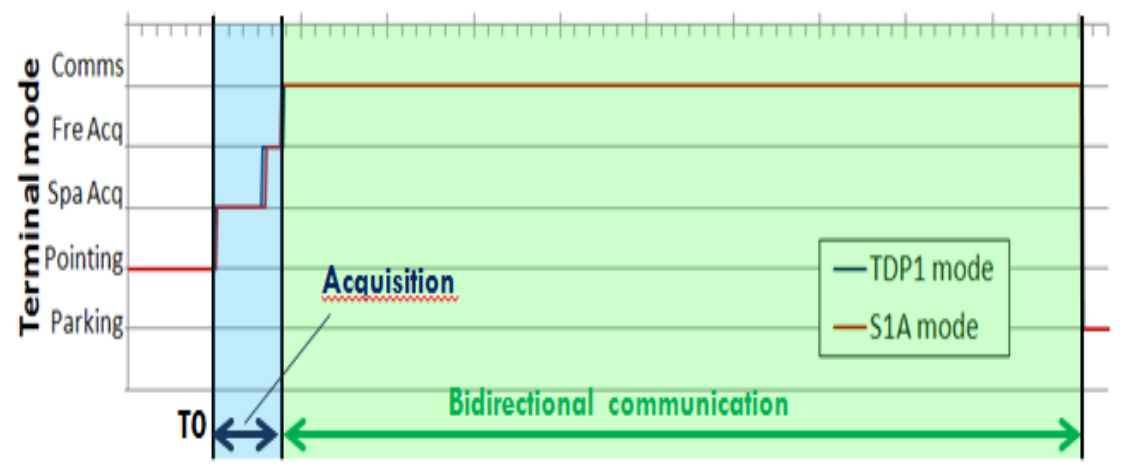

Figure 7: Terminal modes transition during a link along-track movement of the satellites during the signal travel time.

Accurate optimisation of all the parameters involved permits error free optical communication, as shown in fig. 7 , reporting the terminal mode and "User data error count”(ie bit errors before correction on the user data channel of the optical transmission)

\section{Link Termination}

At the end of the (pre-loaded) link duration, both terminals independently terminate communication. They return to "Terminal Ready" mode, ready to start a new link by directly going to the next "link start position", or to return to the "safe" parking position should this be required. 


\section{Alphasat TDP1 \& Sentinel-1A: the first optical partners in orbit}

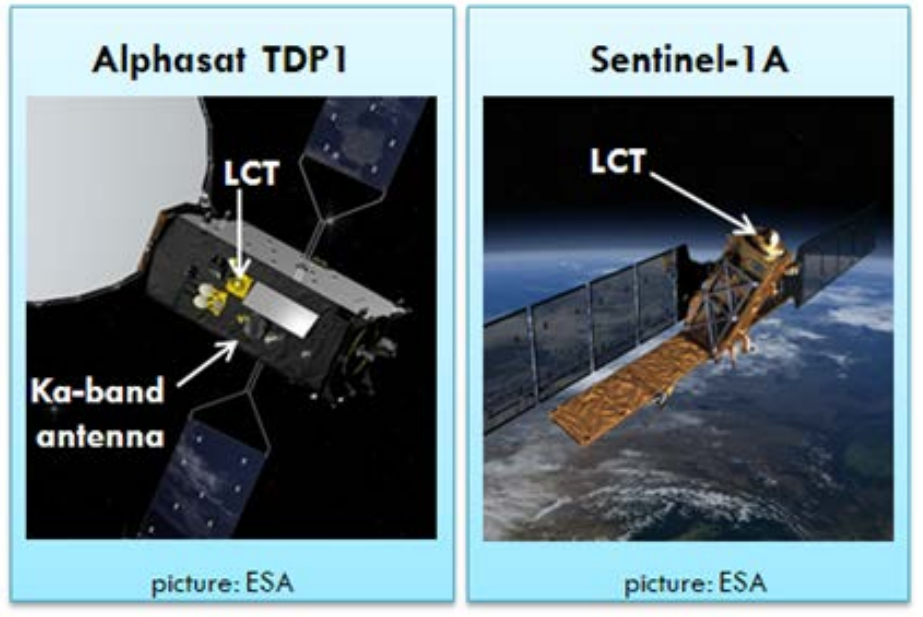

Figure 8: LCTs on Alphasat and Sentinel-1A
Launched in July 2013, owned and operated by Inmarsat plc. of London, Alphasat is the first satellite to be launched using the new European high-power telecommunications platform known as Alphabus, jointly developed by Airbus defense and space and Thales Alenia Space and initiated by a partnership between ESA and CNES.

In additions to the primary mission goal of providing mobile communications as part of the Inmarsat geo-mobile fleet, Alphasat is hosting 4 payloads aiming at in flight demonstration of new technologies and/or constituting the flight segment of specific experiments.

Amongst these "technology demonstration payloads”, TDP1, developed by TESAT for DLR, represented the world's

first available high-speed optical GEO-relay system, consisting of a LCT and the Ka-Band RF downlink transmitter with link capability at data rates up to $1.8 \mathrm{Gbps}$.

Since completion of its in orbit commissioning, TDP1 has used the ESA Optical Ground Station (OGS) of Tenerife (Canary Islands, Spain) to characterize the optical link performances and optimise its configuration parameters, while awaiting the launch of a proper companion in orbit for performing its primary inter-satellite optical link demonstration mission.

Launched in April 2014, Sentinel-1A is the first European satellite contributing to the Copernicus program. Procured and operated by ESA on behalf of the European Commission, it performs its earth observation mission from its heliosynchronous LEO orbit thanks to a Synthetic Aperture Radar. As an additional payload and part of an effort to complement and improve the standard data downlink strategy with an innovative inter-satellite solution, an "Optical Communication Payload” (OCP), based on a LCT identical to the one on Alphasat has been embarked onboard Sentinel-1A.

In November 2014, the first optical link between Alphasat and Sentinel-1A was established. Since then, a large number of links have been performed, demonstrating performance and reliability, and validating system budgets in orbit, meeting the

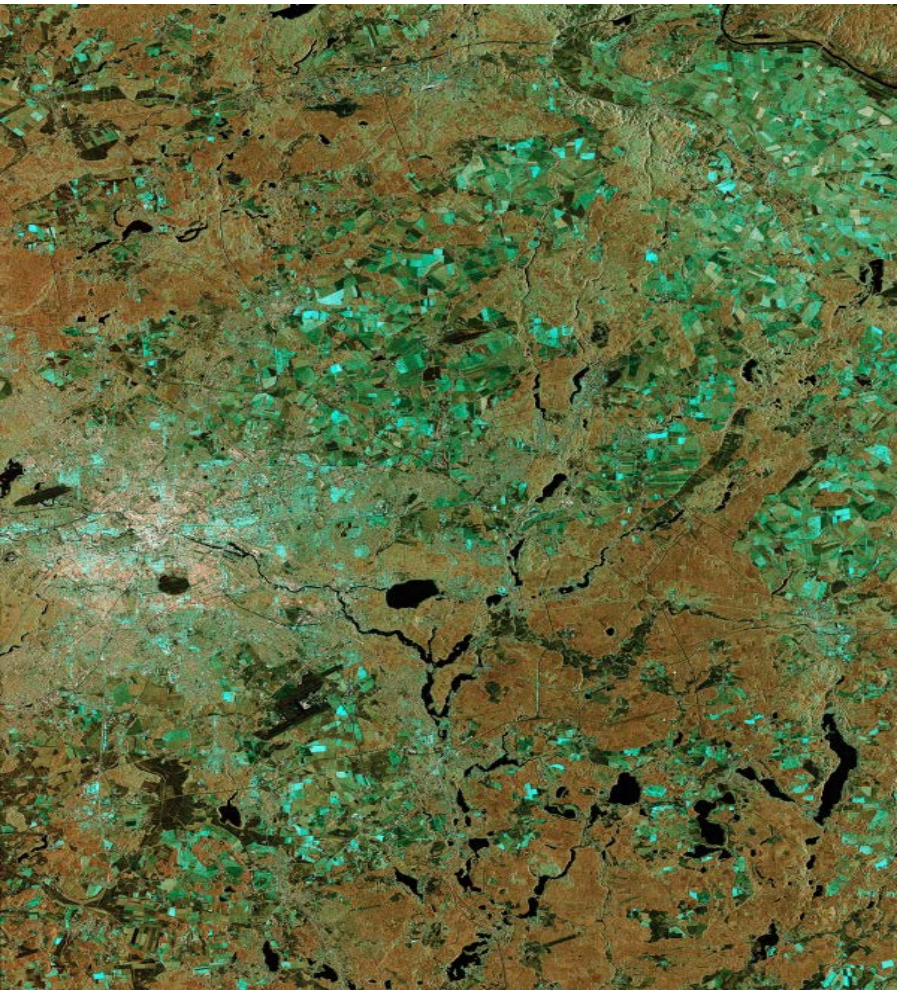

Figure 9: (first) Sentinel-1A SAR image (Berlin, Germany), transmitted via laser requirements of a commercial relay service. Performance details are provided below. 


\section{Alphasat (ASA) and S1A OISL Operations}

\section{General OISL Operations}

In the most generic case, as already mentioned in Sec. III above, the execution of an Optical Inter-Satellite Link implies an exchange of information and coordination for the preparation and commanding of the link. Fig. 10 visualises this generic operations scenario. Although apparently complex, a clear interface definition between the different actors renders the system operations relatively simple.

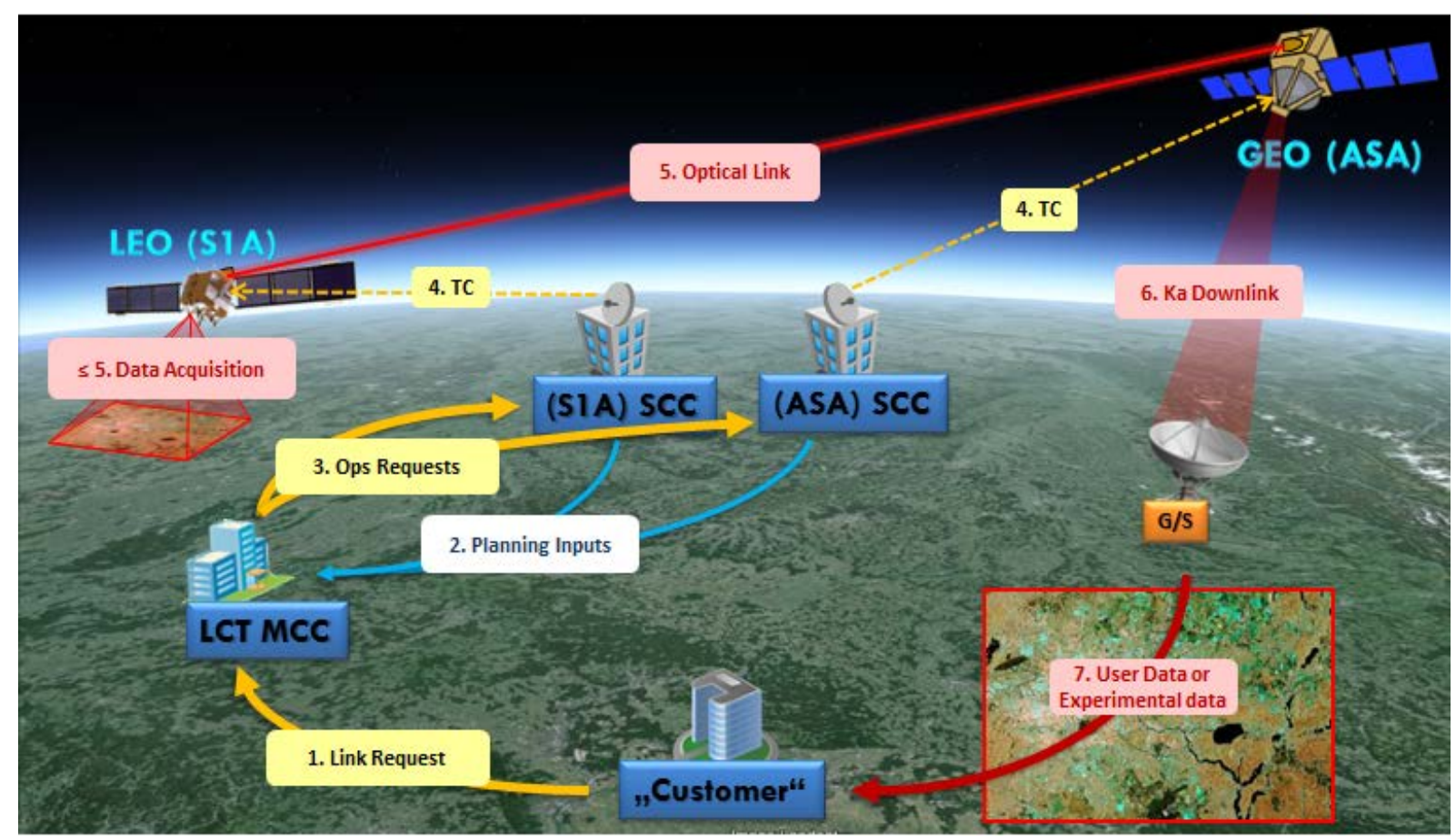

Figure 10: Generic link operations workflow visualisation

The "Customer" provides the initial Link request to the "LCT Mission Control Centre", and receives the "User Data" (step 1 and 7 respectively). The LCT-MCC, based on the planning inputs received by the Satellites Control Centres (SCC, step 2) compiles the "Ops Requests" and delivers them to the corresponding SCC (step 3). The two satellites' SCC, independently command the flight segment (and prepare the ground stations) for the execution of the link (step 4). At the defined link execution time, the OISL takes place (step 5), relying the user data acquired at or before the link execution time (step $\leq 5$ ). During the link itself, the data transmitted on the optical inter-satellite segment is downlinked to the involved GS (step 6). Finally, the data are delivered to the "customer" from the GS (step 7).

For the specific case of ASA and S1A experimental links, the "Customer" shall be replaced by the LCT experiment operations team at TES, and the user data are composed of actual scientific data (S1A SAR images) as well as by actual performance parameters acquired by the LCT during the exercise, and a pre-defined relevant subset of satellite's Housekeeping parameters.

\section{TDP1 Operations on board Alphasat}

As stated above, TDP1 is one of four hosted payloads on the Inmarsat operated Alphasat geostationary communication satellite. As such, the concept of operations has to satisfy the basic requirement of noninterference with the main mission (commercial) objective. To achieve this, the integration of TDP1 in the Inmarsat fleet operations work-flow has been pursued and achieved thanks to an automated operations scenario coordinated by the "TDPs ESA Coordination Office" (TECO).

The weekly planning cycle begins with the availability of (preliminary and final) operations planning input by Inmarsat (platform operational "exclusion periods" and orbital information) that are used by the TDPs operations centres to produce their operations requests. These are de-conflicted and harmonised by the TECO planning system to produce a unique TDP Activities Request File (TARF) that is delivered to Inmarsat. This 
request file is imported directly into the activity schedule from where, at the time indicated in the request, it is initiated and the corresponding automatic execution procedure(s) started. Although not nominally foreseen, INM SCC is able to intervene at run time in case of need.

For TDP1, the experiment operations team at TES compiles the ops request and delivers them to a LCT Mission Control Centre located in DLR-GSOC (German Space Operations Centre). It is here that all (orbital) information regarding TDP1 possible link partners (ground or space-based counter-terminals) are collected and processed. The requests are checked against the system internal constraints and, notably in case of links planning, complemented with the necessary flight dynamics elements (eg the Chebychev polynomials parameters, see section III above). The GSOC MCC finally compiles and delivers to TECO the weekly TDP1 "Input Operations request" and corresponding Procedure Parameters Files (PPF).

It shall be noted that, although the Input Operation Request (IOR) (and TARF) is nominally delivered weekly, the "Procedure Parameters File" associated with each activity scheduled can be updated up to 2 hours before the task execution, thus providing the possibility to specify "fresh" procedures' executions parameters.

For monitoring purposes, a dedicated server of the INM MCS has been customized to provide remote access to Real-Time Telemetry to the TDP operations teams, both via an UDP streaming of the raw TM, as well as directly using the TM de-commutation and visualization capabilities of INM MCS. Daily delivery of both TM and operations ancillary data, as well as a TM archive access on demand are in place to complement the RT monitoring tools and fulfill the needs in terms of data provision to carry out the (experimental) mission of each TDP.

\section{OCP Operations on board Sentinel-1A}

The (heliosynchronous) Sentinel-1A mission calls for a high degree of autonomy with reduced ground centre intervention, so that commanding by ground with immediate execution is minimized. For OCP operations, the spacecraft supports an on-board Mission Timeline (MTL), where the activities are scheduled with respect to time. An Orbit Position Schedule (OPS) is also provided on Sentinel-1A for scheduling activities with respect to orbit position.

The GMES Sentinels Ground Segment is in charge of the overall commanding and monitoring of the various spacecraft constellations (thus including Sentinel-1A) as well as the acquisition, processing and dissemination of their observational data. The two primary components of the Ground Segment are the Flight Operations Segment (FOS) and the Payload Data Ground Segment (PDGS).

Each working day, the PDGS delivers an input plan (Nominal Payload Planning File) covering by default a period of 12 days corresponding to one repeat cycle. If necessary PDGS will modify the plan before distribution to the FOS, e.g. incorporating data "take" requests resulting from user orders submitted since the last update.

The FOS processes the received inputs, generating a schedule covering the complete planned interval (e.g. 12 days), but only uploads commands covering the next 96 hours to comply with the spacecraft on-board queue size limits. As soon as the on-board resources are available, the FOS "tops-up" the command queue by releasing an additional 24 hours' worth of commands, thus ensuring that a plan covering between approx. 72 and 96 hrs is always available on board. This strategy avoids the need for real-time commanding of the payload or subsystems other than unforeseen special operations or recovery activities.

OCP nominal operations shall be conducted as per the main Sentinel-1A payload according to the concept above. In particular, the predicted orbit file and the "Skeleton Plan File" (SPF) are provided by the Flight Dynamic System (FDS). The orbit file, together with orbital information of the counter-terminal hosting satellite (provided by the operator of this latter) are used by PDGS to compile the LCT plan. The SPF, LCT Plan, together with the Operation Planning File (OPF) provided by the Flight Control Team are fed for harmonization in the Mission Planning System, that creates the Plan Increment File and On-Board and Ground Telecommand (TC) schedules.

For the (experimental) activities carried out with ASA-TDP1, the LCT Plans provided by PDGS are substituted by "Special Operation Requests" delivered directly by the OCP experiment operations team of Tesat (this is the same as for TDP1). Also similar to the TDP1 case, in order to prepare these SORs, the SPF and orbit file are distributed to the LCT Mission Control Centre in DLR-GSOC for the preparation of all relevant operational products (notably, link parameters). It shall be noted that the Chebychev polynomials for a link are calculated directly at Sentinel-1A FOS after a link request is received. This implies distribution to the FOS of the counter-terminal orbit information. 
Monitoring of S1A OCP takes place at the dedicated control centre located at ESA-ESOC. Off line access to the relevant TM and ancillary data is provided to TES for performance analysis, based on the External Data Distribution System (EDDS), where data is routed after each TM downlink pass.

\section{Joint S1A\&ASA OISL Operations}

Given the experimental nature of the activities conducted, a joint concept has been agreed for the ASA-S1A Optical Inter-Satellite Links operations, based on the two missions' planning cycle and operations execution scenario. Figure 12 illustrates the concept and work-flow.

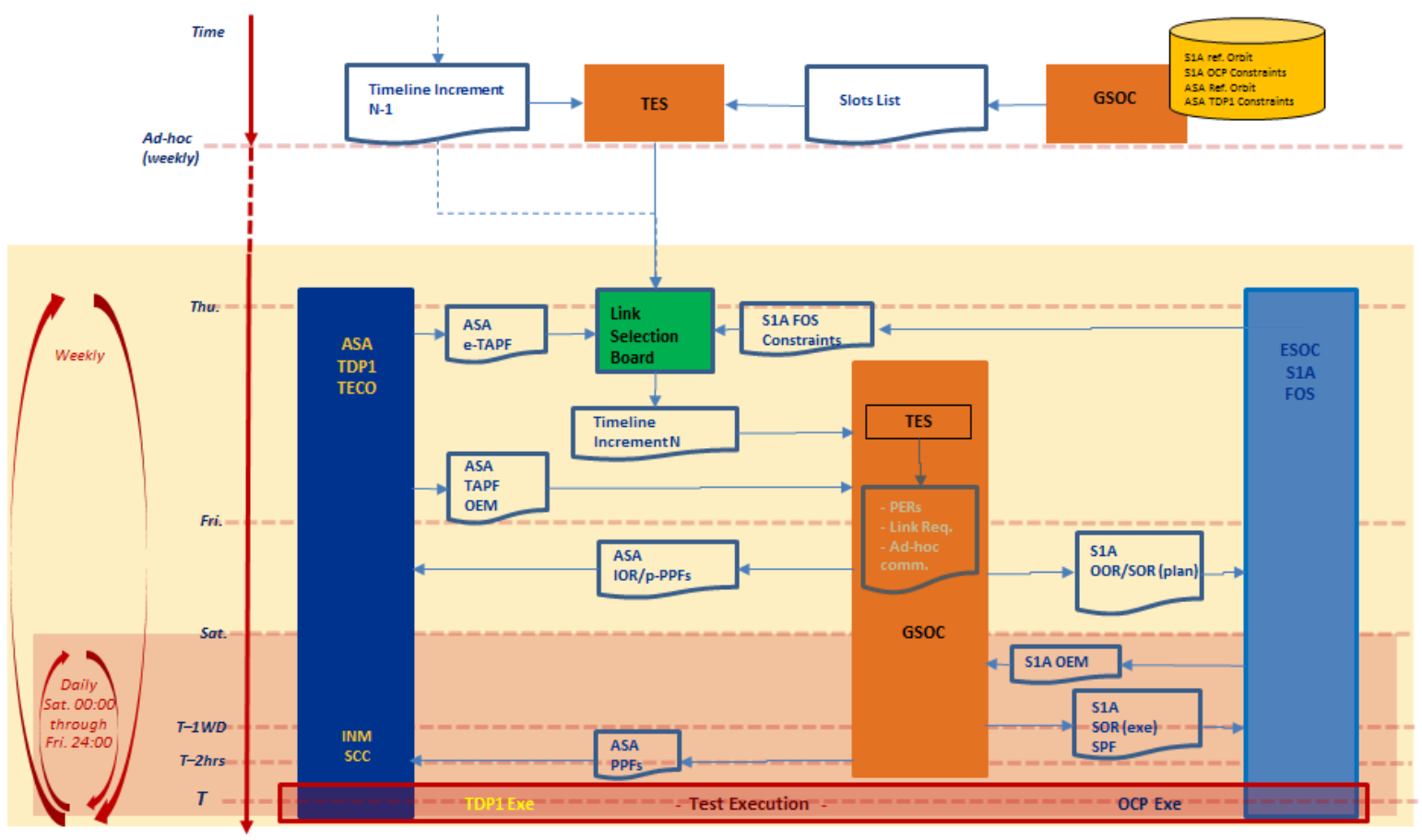

Figure 12: ASA and S1A Joint planning and operations workflow for OISL execution

During the execution of increment N-1, the LCT MCC at GSOC provides the next increment "Slot List", based on the orbits of the two LCT hosting satellites and the terminals operational constraints (both internal and dictated by the hosting mission). Based on this "Slot List" , the LCT Operations Team at TES selects the interesting opportunities to execute links during increment N, and proposes them to the "Link Selection Board" (LSB). The LSB discuss the links proposal in the light of the additional dynamic planning constraints reported in the ASA “enhanced-TDPs Activity Planning File” (e-TAPF) made available by the ASA INM satellite control team and by the S1A FOS. The output of the weekly LSB is the Timeline for increment N, that the LCT Operations team and LCT MCC (TES and GSOC -see above-) translate into the relevant IOR entries and PPFs for ASA, and SORs for S1A.

This simple concept has been permitting an effective and easy coordination of the several actors involved in the links planning and execution, while maintaining the needed independence of each mission's activities.

Moreover, thanks to the unique coordination point in the process represented by the LSB, consistent visibility of the on-going activities is provided also to all stake-holders not directly involved with an active role in the OISL operations.

\section{Link Performance}

Alphasat TDP1 GEO-relay has executed up to the present date more than 200 successful links of both quasioperational and experimental scope. 
During optical link commissioning between Alphasat TDP1 and Sentinel-1A, the total uncertainty cone was initially set to $2250 \mu \mathrm{rad}$ and reduced to $1000 \mu \mathrm{rad}$. During all links, pointing accuracy was found better than $500 \mu$ rad for both link partners. Excellent host attitude knowledge is provided by the Jena-Optronik ASTRO APS star sensor embarked on Alphasat as TDP6.

In parallel to the uncertainty cone, also spatial acquisition duration was reduced from 240s at the very first tests to 34s for default links. During dedicated experimental links, spatial acquisition was reliably achieved within $7 \mathrm{~s}$. Even during links with intentionally adverse conditions like ultra-low TX laser power of 100mW, spatial acquisition was achieved in no more than 80s. Figure 13 shows the 100th link as a typical example of spatial acquisition within 34 seconds for a link with total uncertainty cone of 1000 $\mu$ rad, and 1.1W optical TX power.

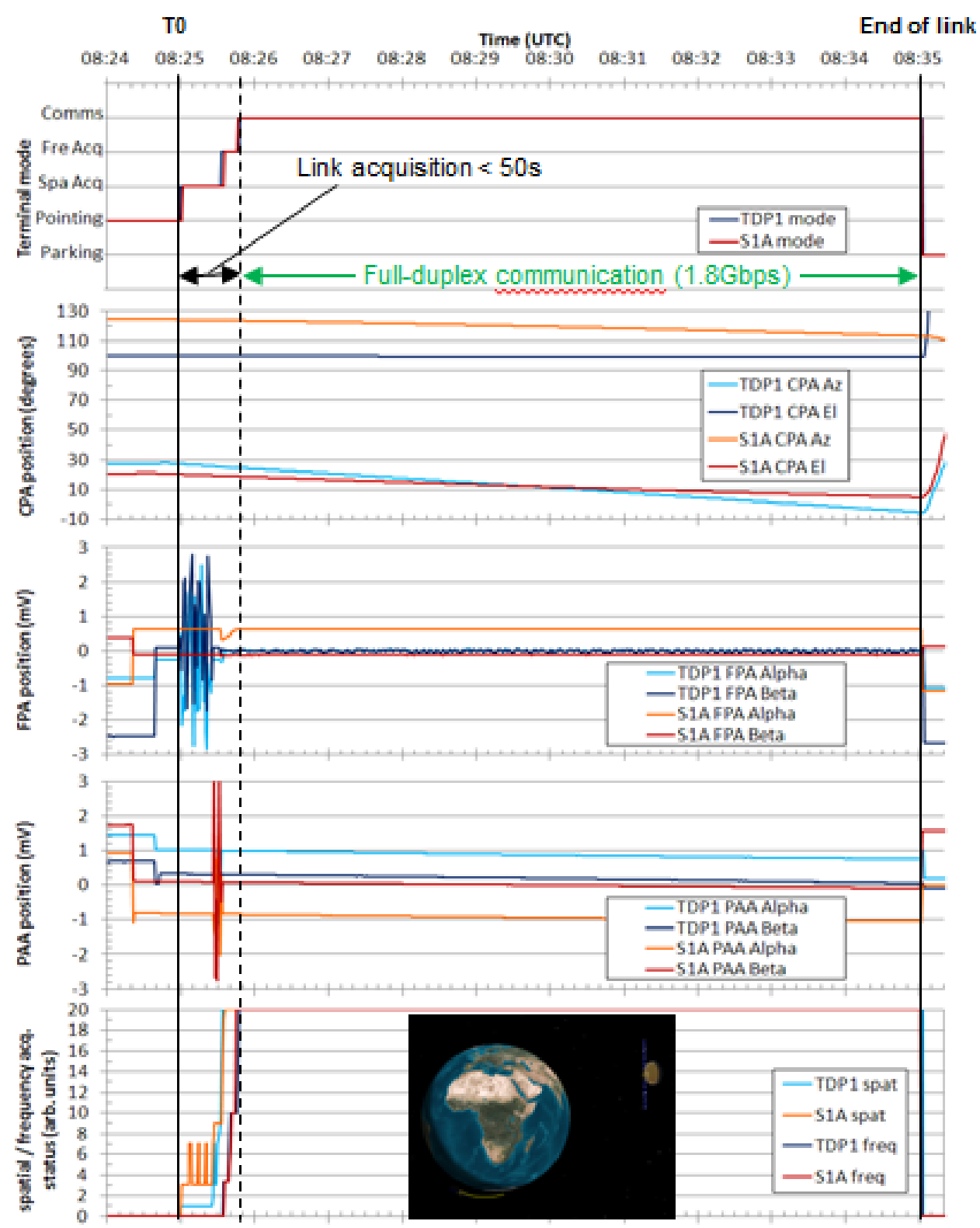

Figure 13: ASA-S1A 100th link - TM shows modes transitions, Coarse and Fine Pointing Asembly positions, Point Ahead Angle position, and Spatial and Frequency Acquisition status. For the successful 10 mins link.

Link acquisition between Alphasat TDP1 and Sentinel-1A is reliably and repeatability achieved within less than 50 seconds after T0. During some specific link sessions, acquisition durations of only 20s have even been 
demonstrated (Figure 14). No impact on acquisition duration could be found due to satellite-induced microvibrations environment.

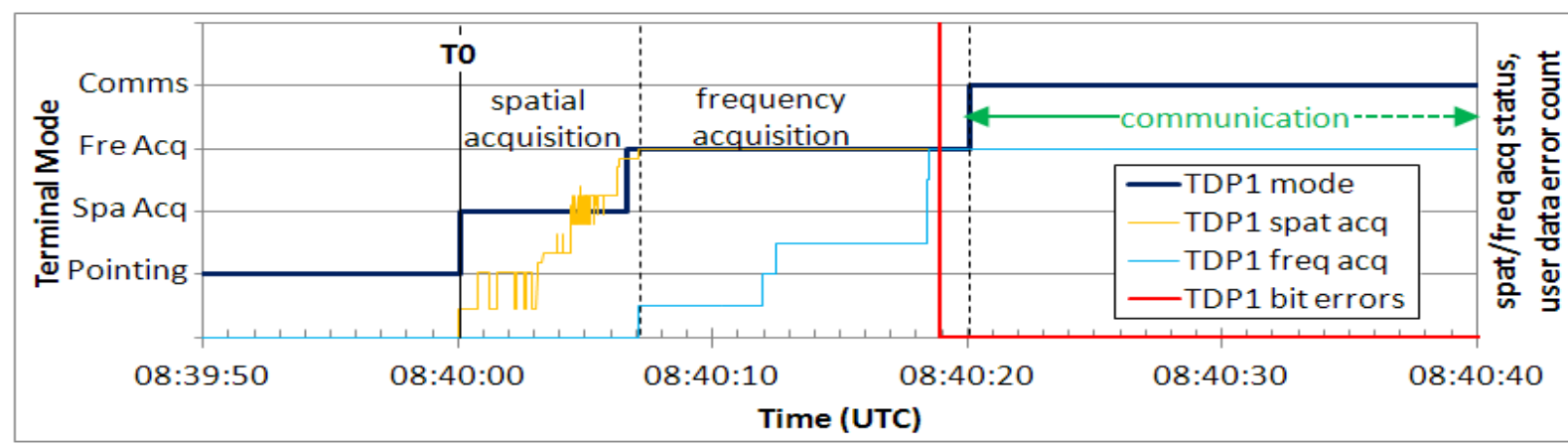

Figure 14: Acquisition within 20s, spatial acquisition 7s. TM recorded at $100 \mathrm{~Hz}$ sampling rate. Only TDP1 is shown (link performed on Aug. 18th, 2015)

During communication, a built-in LPC error detection and correction algorithm ensures integrity of transmitted data even at low signal levels. Using 1.1W optical TX power, during a large number of links not

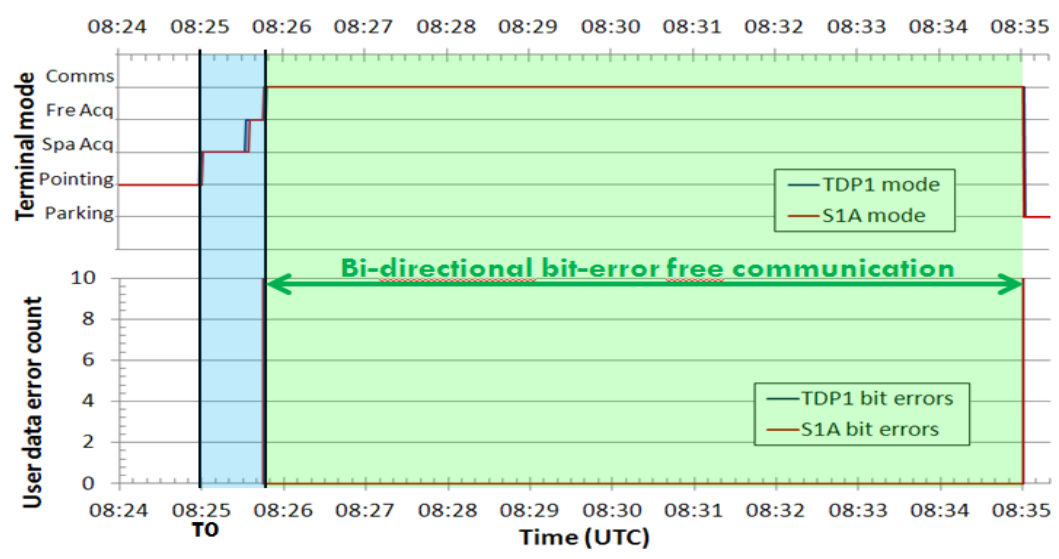

Figure 15: Typical bit errors count for a 10 mis link showing error free data transmission over OISL even a single user data bit error has been recorded. This correlates to a user data bit error rate of much lower than $10^{-12}$. Additional Reed-Solomon encoding of the user data provide further margins.

Figure 15 reports the characteristic signatures of the LCT modes transition and user data bit error recorded on the optical inter-satellite transmission segment of a typical link.

Taking into account the maximum optical TX power of 5W, links with GEO-GEO distance are easily possible with full performance.

\section{Looking ahead, the European Data Relay Satellite System}

As a further in-orbit companion to TDP1, Sentinel-2A, the second European satellite of the Copernicus program was launched in June 2015. It performs its Earth observation mission from its LEO (heliosynchronous) orbit using a multispectral imaging instrument, and, just like Sentinel 1A, carries a LCT to enhance its data return capabilities via optical intersatellite communications.

The first link between Alphasat and Sentinel-2A was performed in September 2015, the details of Sentinel-2A link commissioning will be published elsewhere. Further LCTs

will be embarked onboard Sentinel-1 and Sentinel-2 B/C/D series LEO satellites.

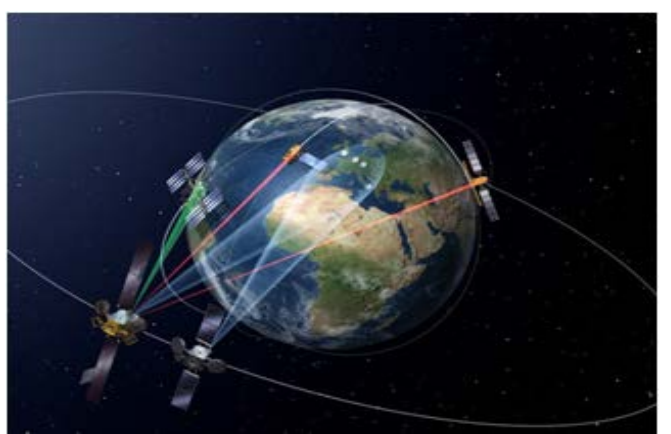

Figure 16: the European Data Relay Satellite System concept (EDRS) 
Alphasat TDP1 serves as the precursor for the forthcoming European Data Relay Satellite System (EDRS). EDRS will eventually consist of a network of dedicated geostationary terminals providing data relay services. The first, EDRS-A as a hosted payload onboard Eutelsat EB9B, has been launched on January, 29th, 2016, and is completing the extensive in-orbit testing campaign necessary to start the active service.

The European Data Relay Satellite System EDRS, will provide commercial GEO data relay service for up to 26 LEO satellites, with EU Copernicus Sentinel-1 and -2 being the first (anchor) clients. EDRS LEO-GEO optical links provide user data rates up to 1.8 Gbps for optical LEO-GEO inter-

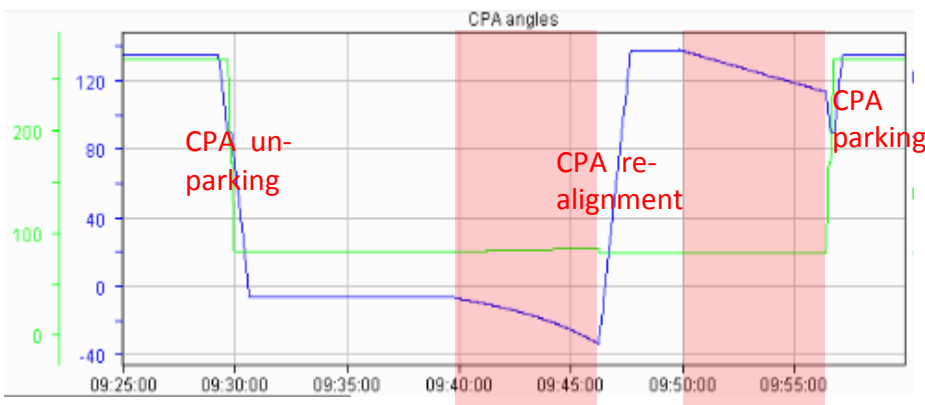
satellite link and Ka-band RF downlink. Each EDRS GEO-relay satellite can downlink data volumes of up to 16.2 TByte per day, paving the way towards the SpaceDataHighway.

EDRS will give the user on the ground high-speed access to real-time Earth Observation data, on-demand at the right place and time. Applications for EDRS service include emergency response, open ocean surveillance, UAS communication, weather forecasting and wide-area monitoring.

Beyond providing the most representative technology demonstration test-bench possible, the operational experience being gained with ASA TDP1 and Sentinel-1A (and Sentinel2A) is proving of great benefit for the full understanding of the various specific issues characterizing Optical InterSatellite Links operations, in particular the operations of the specific TES LCT.

In the light of the benefit and the results obtained, it has been agreed to extend the "experimentation phase" of the first Optical intersatellite link partners (ASA and S1A), beyond the originally planned end of the activities, continuing the operations in parallel with S2A, taking advantage from the new "three optical communications partners" scenario.

Thanks to the availability of Sentinel-2A, the first "dual link” test has been performed on April 7th, 2016, demonstrating the operational and technical feasibility of consecutive links to different targets (without returning the LCT CPA to parking), in a first real-life EDRS service scenario. Figure 17 shows the actual geometry of the links, the TDP1 LCT Coarse and Fine Pointing Assembly angles, and the stable Tracking Sensor signal recorded during the exercise.

\section{Conclusions}

Alphasat TDP1 and Sentinel-1A have successfully demonstrated and validated in orbit the feasibility, reliability and simplicity of an optical GEO-relay to provide high-speed, near-real-time access to the mission data of LEO satellites outside the range of direct RF ground stations.

The links performed have also permitted acquisition of an invaluable experience in the Ground Segment and operations specific features implied by the planning and execution of Optical Inter-Satellite Links. 
Links will continue between Alphasat and both Sentinel-1A and Sentinel-2A. The system is the precursor mission for the European Data Relay Satellite System, currently under deployment, which is expected to start commercial service in 2016.

\section{References}

${ }^{1}$ ESA Telecommunication and Integrated Application, “ARTES8”,URL: http://telecom.esa.int/telecom/www/area/index.cfm 2 D. Troendle, C. Rochow, P Martin Pimentel, H. Zech, F. Heine, H. Kaempfner, M. Gregory, M. Motzigemba, U. Sterr, R. Meyer, M. Lutzer, S. Philipp-May, “Optical LEO-GEO Data Relays: From Demonstrator to Commercial Application”, AIAA Space and ICSSC 2014

${ }^{3}$ G. Muehlnikel, H. Kaempfner, F. Heine, H. Zech, D. Troendle, R. Meyer, S. Philipp-May, „The Alphasat GEO Laser Communication Terminal Flight Acceptance Tests“, ICSOS 2012

${ }^{4}$ F. Heine, G. Muehlnikel, H. Zech, D. Troendle, S. Seel, M. Motzigemba, R. Meyer, S. Philipp-May, E. Benzi, „LCT for the European data relay system: in orbit commissioning of the Alphasat and Sentinel 1A LCTs “, Proc. SPIE 9354, 2015

${ }^{5}$ M. Witting, H. Hauschildt, A. Murrell, J-P. Lejault, J. Perdigues, J.M. Lautier, C. Salenc, K. Kably, H. Greus, F. Garat, H.L. Moeller, S. Mezzasoma, R. Meyer, B. Guetlich, S. Philipp-May, A. Pagels-Kerp, B. Theelen, M. Wiegand, M. Leadstone, G. Eckert, G. Wuetschner, L. Laux, O. Gerard, D. Poncet, R. Mager, K. Schoenherr, F. Heine, S. Seel, K. Panzlaff, H. Zech, H. Kaempfner, A. Schneider, I. Gutierrez Canaz, C. Arias Perez, H. Schuff, "Status for the European Data Relay Satellite System”, ICSOS 2012

${ }^{6}$ E. Benzi, A. Cacioni, "Private Public Cooperation for Hosted Payload Operations: the Alphasat Concept”, AIAA SpaceOps 2014 Conference, May 2014

${ }^{7}$ E. Benzi, N. Policella, M. James, A. Cacioni, “Alphasat Mission: TDPs Ground Segment and Operations Concept \& Implementation”, 20th Ka-band Conference, Oct. 2014

${ }^{8}$ Marina Bernard, Edoardo Benzi, Juan Rivera Castro, Philippe Sivac, Eloy Torres, Simon Weinberg, “Alphasat: Innovation in Orbit”, ESA Bulletin 159, Aug. 2014

${ }^{9}$ P. Bargellini, P.P. Emanuelli, I. Shurmer, F. Marchese, C. Steiger, H.L. Moeller, “The GMES-Sentinels Flight Operations Concept”, AIAA SpaceOps-2012 Conference, June 2012

${ }^{10} \mathrm{http}: / /$ www.edrs-spacedatahighway.com/ 